\title{
Major antiseptic in dentistry
}

\author{
Shaik Ali Hassan ${ }^{1 *}$, Sumit Bhateja ${ }^{2}$, Geetika Arora ${ }^{3}$, Francis Prathyusha ${ }^{4}$ \\ ${ }^{1}$ Dental Surgeon, ${ }^{2} \mathrm{HOD},{ }^{3}$ Reader, ${ }^{4} \mathrm{BDS}$, MDS, Dept. of Dental, ${ }^{1,4}$ Francis Maxillofacial and Dental Clinic, India, ${ }^{2}$ Manav \\ Rachna Dental College, Faridabad, Haryana, ${ }^{3}$ Inderprastha Dental College \& Hospital, Ghaziabad, Uttar Pradesh, India
}

*Corresponding Author: Shaik Ali Hassan

Email: alishaikhassan@gmail.com

\begin{abstract}
Over the years chlorhexidine has been used in dental practice as an excellent antiplaque agent. Chlorhexidine (CHX) not only shows the special properties of substantivity but, also has a broad spectrum antimicrobial activity that makes its use in various oral disorders. Almost all disciplines of dentistry make use of this material in different formulations such as mouth wash, gel, varnishes and restorative materials etc.
\end{abstract}

Keywords: Chlorhexidiene, Root canal irrigant, Phenols, Apthous stomatitis.

\section{Introduction}

It is an established fact that dental caries and periodontal disease are the two predominant diseases affecting the oral cavity and dental plaque plays a key role in the progression of these two diseases. Dental plaque forms naturally on the teeth and in the absence of adequate oral hygiene, it can accumulate beyond the levels that are compatible with dental health and at susceptible sites dental caries or periodontal disease or both can occur. Effective removal of dental plaque is one of the main strategies for the prevention of these two diseases. Plaque control by mechanical debridement is highly labour intensive whether professionally administered or practiced personally, satisfactory home care further demands a measure of manual dexterity and a high degree of motivation, which many individual do not possess. Not surprisingly, a large number of chemical agents have been tested for their ability to reduce plaque accumulation. Although many antimicrobial agents would appear to be suitable for plaque control, only few have been found to possess clinical efficacy. This is because many of the antimicrobial agents do lack property of substantivity and lacks efficacy against oral microorganisms. Currently formulated antimicrobial agents include essential oils, metals (zinc, stannous, copper), phenols (triclosan), plant extracts, (Terminalia chebula extract, garlic extract. occimum sanctum, triphala, aloe vera enzymes etc. ${ }^{1-4}$

Chlorhexidine is a bisbiguanide formulations with cationic nature. symmetric molecules with two chlorophenyle and two rings linked by a group bigunide central hexamethylene chain. This is a solid foundation and the most stable in salt form. The most common preparation is digluconate salt because of its water solubility. Chlorhexidine was developed in the 1940s as a result of search for antiviral agents. Chlorhexidine found that does not have antiviral activity but instead it has antibacterial activity. The use of chlorhexidine started as general disinfectant with a broad antimicrobial spectrum. Antimicrobial spectrum, including most of the microbes such as gram-positive and gram-negative organisms including bacteria spores, lipophilic viruses, yeasts and dermatophytes etc.,6 Chlorhexidine is widely used in various medical fields such as gynecology, urology, ophthalmology and treatment of burns etc. The first use of chlorhexidine in the dental practice is the site washing and disinfecting root canal surgery. ${ }^{7-9}$

\section{Chlorhexidine on plaque}

Several studies have proven efficacy in vivo and in vitro $0.2 \%$ chlorhexidine as antiplaque agents. ${ }^{9-16}$ Effect from chlorhexidine on plaque inhibition is dose dependent, the dose typically range in concentration from 0.03 to $0.2 \%$ volume, frequency and concentration are important in determining clinical response. The optimum dose of chlorhexidine as gargle generally considered to be $20 \mathrm{mg}$ twice daily, the same level of plaque inhibition could be achieved with greater volume lower concentrations. A lower concentration of chlorhexidine has been tested in several studies and proved effective. A persistent bacteriostatic action lasting for 12 hours was observed. There is no significant difference in plaque when $0.2 \%$ chlorhexidine mouthwash used for 15,30,60 seconds. There was no difference in plaque inhibiting action of $0.1 \%, 0.12 \%$ and $0.2 \%$ of chlorhexidine rinse. ${ }^{17-22}$ Bonesvoll in his book studies reported that there is a rapid binding of chlorhexidine onmouth during the first 15 seconds of rinsing and almost $75 \%$ after 30 seconds of rinsing. ${ }^{23}$ However invitro studies recently have 
shown a $0.12 \%$ chlorhexidine has The antibacterial activity in both planktonic and biofilm organisms. ${ }^{24}$ The substantivity of chlorhexidine is associated with controlled release system. The presence of $\beta$ cyclo dextrin regulates and controls the amount of $\mathrm{CHX}$ released. Greater the amount of $\beta$ cyclo dextrin, more progressive CHX release. The development of controlled release system of cellulose substrates can also be achieved by using microfibrillated cellulose (MFC). A new trial the approach proposed for the development of bio-based controlled release system. $\beta C D$ and MFC mixed together to create synergies between their ability to control release the active molecule. MFC Association and $\beta C D$ gives very promising results. The obtained release pattern has a combination of both actions MFC and $\beta C D$. MFC mainly act on the effects of the explosion, while $\beta C D$ controlled and $\mathrm{CHX}$ regulated release from time to time. Therefore, complementary measures can be achieved by connecting the two release system. Depending on the needs of end users, CHX system / MFC / $\beta C D$ will release a higher number of $\mathrm{CHX}$ progressive system / $\beta C D{ }^{25}$

\section{Effect of chlorhexidiene on oral tissues}

Some studies support the view that the use of prolonged chlorhexidine is not associated with the development of resistant strains of microorganisms. With Long-term use of chlorhexidine, staining of teeth, the emergence of opportunistic pathogens or steady shift in oral flora has been reported. ${ }^{1}$ Clinical studies indicate that the use of $0.2 \%$ chlorhexidine mouth wash causes a reduction in the number of oral bacteria without overgrowth by candida albicans or E. coli. A number of studies have examined the ability to produce oral bacteria resistant to chlorhexidine in the laboratory.

\section{Chlorhexidine and Its Use in HIV Infection}

Common oral disease such as gingivitis and periodontitis are usual in HIV patients. Palliative therapy for these conditions can prevent the more serious complications. Chlorhexidine plays an important adjunctive role in the treatment of HIV associated gingivitis and periodontitis. apthous stomatatis, candidiasis, herpes virus and HIV associated neoplastic lesions. Chlorhexidine found to be effective in reducing candida species in HIV affected individuals and children. ${ }^{26,27}$

\section{In Patients with Drug Induced Gingival Enlargement}

Chlorhexidine has an adjunctive role in the treatment of drug induced gingival enlargement. ${ }^{28}$ The overall effect is not known as the research is inadequate and consist of mixed reports. More research is needed to evaluate the effect of chlorhexidine on the inflammation associated with gingival enlargement.

\section{Use of Chlorhexidine in Recurrent Apthous Stomatitis}

Chlorhexidine can be used in patients suffering from recurrent apthous ulceration on the basis that natural course of recurrent oral ulcers can be extended due to bacterial contamination. Several studies support the benefits of this therapy but chlorhexidine mouth rinse is of limited or no effect on established major apthous ulceration. ${ }^{29}$ Studies have shown that chlorhexidine mouth rinse can reduce the incidence, severity and duration of apthous ulceration whereas chlorhexidine gel significantly reduced severity and duration but not incidence. ${ }^{30}$

\section{Chlorhexidiene in dry socket}

A significant reduction in the incidence of dry socket is observed in studies Larsen et al. ${ }^{31}$ Hedstorm L 2007, found no effects on alveolar osteitis reduction. ${ }^{32}$ More recently, a $0.2 \%$ bioadhesive gel form has become available and is more effective than the mouth wash. Its main advantage is that it extend the bioavailability of chlorhexidine in the application area. The topical application of chlorhexidine gel bioadhesive for surgical wound for a week after surgery possibly decreases the incidence of alveolar osteitis after extraction of mandibular third molar. ${ }^{33}$ Babar A reported that one application of chlorhexidine gel effectively reduce the frequency of alveolar osteitis. ${ }^{34}$ It is seen that increased concentartion from $0.2 \%$ to $1.2 \%$ gel is not possible as it doesn't show much affect. ${ }^{35}$

\section{As a root canal irrigant}

Intracanal tissue treated with chlorhexidine really inhibits the growth of E. feacalis. Martin and Nind investigating the efficacy of chlorhexidine as a disinfectant in presurgical apicectomy site observed the beneficial effects. ${ }^{36}$ Numerous studies have prove that $2 \%$ chlorhexidine as effective as $5.25 \%$ sodium hypochlorite in reducing the growth of E.feacalis..$^{37-45}$ With a high concentration of substantivity of chlorhexidine was found for 12 weeks. The antimicrobial activity of $\mathrm{CHX}$ is reduced when combined with other substances, including $\mathrm{CH}, \mathrm{CH}$ plus zinc oxide, among others. ${ }^{46-50}$ However, CHX alone does not act as a physical barrier and does not present radiopacity. The use of $\mathrm{CHX}$ gel as intracanal medicament is recommended for a short period of time (3-5 days), particularly in those cases where the canals 
were fully instrumented but could not be root-filled due to the lack of time. It is also recommended in cases of exudation, as it retains its antimicrobial activity in the presence of blood and other organic matters. ${ }^{51,52} \mathrm{CHX}$ gel is delivered into the canals with a syringe with 24gauge needle, being easily introduced and removed from the root canals.). ${ }^{53-57}$ Dornellis-morgental study observed that chlorhexidine irrigation solution can prevent the activity but did not eradicate E. faecalis in the root canal system. ${ }^{58-60}$

\section{Conclusion}

Chlorhexidine is not only an excellent antiplaque agent but also has excellent antimicrobial properties. Its extensive antimicrobial spectrum can be regarded as a benefit for maintaining overall oral health. A wealth of research supports its use in a variety of forms and in a variety of oral disorders. Although its use is limited because of side effect, new formulations with anti discolouration system has shown promising results. More importantly chlorhexidine has shown promising results in controlling dental caries.

\section{Source of Funding}

None.

\section{Conflict of Interest}

None.

\section{References}

1. Walker CB. Microbiological effects of mouth-rinses containing antimicrobials. J Clin Periodontol 1988;15:449505.

2. Mandel ID. Antimicrobial mouthrinses: Overview and update. J Am Dent Assoc 1994;125(2):2S-1OS.

3. Pitten FA, Splieth C, Kramer A. Prophylactic and therapeutic application of antimicrobial agents in the oral cavity. Pharmazie 2000;55:635-9.

4. Gupta D, Guptab RK. Dara John Bhaskar. Vipul Guptad:Comparative Evaluation of Terminalia chebula Extract Mouthwash and Chlorhexidine Mouthwash on Plaque and Gingival Infl ammation - 4-week Randomised Control Trial. Oral Health Prev Dent 2015;13:5-12.

5. Jone $\mathrm{C}$ G. Chlorhexidine is it still a gold standard. Periodontol 2000;15:55-62.

6. Denton G W. Disinfection, sterilization and preservation.1991, 4th edn. 274-289.

7. Lim KS. Chlorhexidine pharmacology and clinicl applications anaesthetic intensive care. 2008;36:50-12.

8. Fardal O, Turnbull RS: A review of the literature on use of chlorhexidine in dentistry. J Am Dent Assoc 1986;18:863-9.

9. Johnson BT. Uses of chlorhexidine in dentistry. General Dent 1995;43:126-32.

10. Hull P S. Chemical inhibition of plaque. J Clin Periodontol 1980;7:431-42

11. Davies RM. The effect of topical application of chlorhexidine on the bacterial colonization of the teeth and gingiva. Period Res 1970;5:96-101.
12. Flotra, L, Gjenno P, Rolla G. A 4-month study on the effect of chlorhexidine mouth washes on 50 soldiers. Scand J Dent Res 1972;80:10-7.

13. Gjermo P, Rolla G. Plaque inhibition by antibacterial dentifrices. Scand J Dent Res 1970;78:464-70.

14. Bonesvoll P, Lokken P, Rolla, G. Influence of concentration, time, temperature and $\mathrm{pH}$ on the retention of chlorhexidine in the human oral cavity after mouth rinses. Arch Oral Biol 1974;19:1025-9.

15. Flotra L, Gjenno P, Rolla G. \& Waerhaug J. A 4-month study on the effect of chlorhexidine mouth washes on 50 soldiers. Scand J Dent Res 1972;80:10-7.

16. Loe H, Schiott CR. The effect of mouth rinses and topical application of chlorhexidine on the development of dental plaque and gingivitis in man. J Period Res 1970;5:79-83.

17. Segreto VA, Collins E. IVl, Beiswanger BB. A comparision of mouthrinses containing two concentrations of chlorhexidine. J Period Res 1986;21:23-32.

18. J Ernst C. P, Prockl K, Wil Jershausen B. The effectiveness and side effects of $0.1 \%$ and $0.2 \%$ chlorhexidine rnouthrinses: a clinical study. Quintessence Int 1998;29:443-8.

19. Berchier CE, Slot DE, Van der Weijden GA. The efficacy of $0.12 \%$ chlorhexidine mouthrinse compared with $0.2 \%$ on plaque accumulation and periodontal parameters: a systematic review. J Clin Period 2006;33: 561-7.

20. Keijser JA, verkede H, Timmerman MF. Comparison of two commercially available chlorhexidine mouth rinses. $J$ Clin Period 2003;74:214-8.

21. Van Strydonck DA, Timmerman MF, van der Velden U, van der Weijden GA. Plaque inhibition of two commercially available chlorhexidine mouthrinses. J Clin Period 2005;32:305-9.

22. Rath SK, Singh M. Comparative clinical and microbiological efficacy of mouthwashes containing $0.2 \%$ and $0.12 \%$ chlorhexidine. Dent Res J 2013;10:364-9.

23. Sbordone L, Bortoralia C. Oral microbial biofilms and plaque related disease, microbial Communities and their role in the shift from oral to disease. Clin Oral Investig 2003;7:181-8.

24. Babu JP, Garcia-Godoy F. In vitro comparison of commercial oral rinses on bacterial adhesion and their detachment from biofilm formed on hydroxyapatite disks. Oral Health Prev Dent 2014;12:365-71.

25. Lavoine N, Tabary N, Desloges I, Martel B, Bras J. Controlled release of chlorhexidine digluconate using $\beta$ cyclodextrin and microfibrillated cellulose. Colloids Surfaces B: Biointerfaces 2014;121:196-205.

26. Barasch A, Safford MM, Dapkute-Marcus I. Efficacy of chlorhexidine gluconate rinse for treatment and prevention of oral candidiasis in HIV-infected children: a pilot study. Oral Surg Oral Med Oral Pathol Oral Radiol Endod 2004;97:204-7.

27. Ellepola AN, Samaranayake LP.Adjunctive use of chlorhexidine in oral candidoses: a review. Oral Dis 2001;7:11-7.

28. Pilatti GL, Sampaio JE. The influence of chlorhexidine on the severity of cyclosporin A-induced gingival overgrowth. J Periodontol 1997;68:900-4.

29. Addy M, Hunter L. The effects of a $0.2 \%$ chlorhexidine gluconate mouthrinse on plaque, toothstaining and candida in aphthous ulcer patients. A double-blind placebocontrolled crossover study. J Clin Period 1987;14:267-73. 
30. dy. Journal of Clinical Periodontology. 1987; 14: 267-73. 57. Addy M. Hibitane in the treatment of aphthous ulceration. J Clin Period 1977;4:108-16.

31. Larsen PE. The effect of a chlorhexidine rinse on the incidence of alveolar osteitis following the surgical removal of impacted mandibular third molar. J Oral Maxillofac Surg 1991;49:932-7.

32. Hedstrom L, Sjogren P. Effect estimates and methodological quality of randomized controlled trials about prevention of alveolar osteitis following tooth extraction. Oral Surg Oral Med Oral Pathol Oral Radiol Endod 2007;103:8-15.

33. Hita-Iglesias P, Torres-Lagares D, Flores-Ruiz R. Effectiveness of chlorhexidine gel versus chlorhexidine rinse in reducing alveolar osteitis in mandibular third molar surgery. J Am Dent Assoc 1994;125(2):2S-10S.

34. Babar A, Ibrahim MW, Baig NJ. Efficacy of intraalveolar chlorhexidine gel in reducing frequency of alveolar osteitis in mandibular third molar surgery. J Coll Physicians Sur Pak 2012;22:91.

35. Rodriguez - Perez M. Effectiveness of $1 \%$ versus $0.2 \%$ gels in reducing alveolar osteitis from mandibular third molar surgery. Med Oral Patologia Oral y Cirugia Bucal 2013;18:693-700.

36. Martin MV, Nind D. Use of chlorhexidine gluconate for preoperative disinfection of apicectomy sites. Braz Dent $J$ 1987;162:459-61.

37. Dornelles-Morgental R, Guerreiro-Tanomaru JM. Antibacterial efficacy of endodontic irrigating solutions and their combinations in root canals contaminated with Enterococcus faecalis. Oral Surg Oral Med Oral Pathol Oral Radiol Endod 2011;112:396-400.

38. Kuruvilla JR, Kamath MP Antimicrobial activity of $2.5 \%$ sodium hypochlorite and $0.2 \%$ chlorhexidine gluconate separately and combined, as endodontic irrigants. J Endod 1998;24:472-6.

39. Mohammadi Z, Abbott PV. Antimicrobial substantivity of root canal irrigants and medicaments: a review. Aus Endod $J$ 2009;35:131-9.

40. White RR, Hays GL, Janer LR Residual antimicrobial activity after canal irrigation with chlorhexidine. J Endod 1997;23:229-31.

41. White RR, Janer LR, Hays GL.Residual antimicrobial activity associated with a chlorhexidine endodontic irrigant used with sodium hypochlorite. Am J Dent 1999;12:148-50.

42. Zehnder M. Review Root canal irrigants. J Endod 2006;32:389-98.

43. El Karim I, Kennedy J, Hussey D. The antimicrobial effects of root canal irrigation and medication. J Oral Maxillofac Surg 2008;66:441-5.

44. Silva AR, Pinto SC, Santos EB, Santos FA, Farago PV, Gomes JC et al, Pina-Vaz I, Carvalho MF New intracanal formulations containing doxycycline or chlorhexidine against Enterococcus faecalis. J Contemp Dent Prac 2014;15:61-5
45. Van der Vyver PJ, Botha FS, de Wet FA. Antimicrobial efficacy of nine different root canal irrigation solutions. $J$ South Afr Dent Assoc 2014;69:158-60, 162-5.

46. Emilson CG. Potential efficacy of chlorhexidine against mutans streptococci and human dental caries. J Dent Res 1994;73:682-9.

47. K raal, J.H., and $\mathrm{W}$ ong, P.H. C hlorhexidine feeding and its effect on grow th a n d intestinal microflora in rats. Pharm Scol Ther Dent 1978;3(2-4):111-5.

48. Luostarinen V, Effect of chlo rhexidine on the hamster cheek pouch. J Periodontol 1977;48(4):212-5.

49. Harvey BV, Squier CA, Hall BK. Effects of chlorhexidine on the structure and perm eability of hamster cheek pouch mucosa. J P Eriodontol 1984;55(10):608-14.

50. Osmundsen PE. Contact dermatitis to chlorhexidine. Contact Dermatitis 1982;8(2):81-3.

51. Case DE. Safety of Hibitane. Laboratory experiments. $J$ Clin Period 1977;4(5):66-72.

52. G oldschmidt P, Cogen R, Taubman S. Cytopathologic effects of chlorhexidine on hum an cells. J Periodontol 1977;48(4):212-5.

53. K nuuttila, M., and S oderling, E. E ffect of chlorhexidine on the release of lysosom al enzym es from $\mathrm{c} \mathrm{u}$ ltu re $\mathrm{d} \mathrm{m}$ acrophages. Acta Odontol Scand 1981;39(5):285-9.

54. Oydna J, Gjermo P. Effect of chlorhexidine mouthrinses on concentration of IgA in expectorates. Scand J Dent Res 1982;90(3):189-92.

55. Prasad, I. M utagenic effects of the herbicide 3', 4' dichloro propionanilide and its degradation products. Canad J Microbiol 1970;16:369-72.

56. National Cancer Institute, Carcinogenesis. T echnical Support Series no 189, 1979.

57. Kakehashi S, and Parakkal P.I, eds. P roceedings from state of the art workshop of surgical therapy for periodontitis. $J$ Periodontol 1982;53(8):482.

58. Cabral CT, Fernandes MH. In vitro comparison of chlorhexidine and povidone-iodine on the long-term proliferation and functional activity of human alveolar bone cells. Clin Oral Investig 2007;11:155-64.

59. Lee TH, Hu CC, Lee SS, Chou MY, Chang YC. Cytotoxicity of chlorhexidine on human osteoblastic cells is related to intracellular glutathione levels. Int Endod J 2010;43:430-5.

60. Ribeiro DA, Scolastici C, de Almeida PLA, Marques PLA, Marques MEA, Salvadori MF et al. Genotoxicity of antimicrobial endodontic compounds by single cell gel (comet) assay in Chinese hamster ovary (CHO) cells. Oral Surg Oral Med Oral Pathol Oral Radiol Endod 2005;99:637-40.

How to cite: Hassan SA, Bhateja S, Arora G, Prathyusha F. Major antiseptic in dentistry. IP J Surg Allied Sci 2020;2(2):29-32. 